\title{
Shear stress-dependent regulation of apical endocytosis in renal proximal tubule cells mediated by primary cilia
}

\author{
Venkatesan Raghavan ${ }^{a}$, Youssef Rbaibi ${ }^{\mathrm{a}}$, Núria M. Pastor-Soler ${ }^{\mathrm{a}, \mathrm{b}}$, Marcelo D. Carattino ${ }^{\mathrm{a}, \mathrm{b}}$, and Ora A. Weisz ${ }^{\mathrm{a}, \mathrm{b}, 1}$

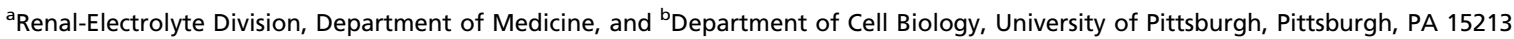

Edited by Marilyn G. Farquhar, University of California, San Diego, La Jolla, CA, and approved May 6, 2014 (received for review February 5, 2014)

\begin{abstract}
The kidney has an extraordinary ability to maintain stable fractional solute and fluid reabsorption over a wide range of glomerular filtration rates (GFRs). Internalization of filtered low molecular weight proteins, vitamins, hormones, and other small molecules is mediated by the proximal tubule (PT) multiligand receptors megalin and cubilin. Changes in GFR and the accompanying fluid shear stress (FSS) modulate acute changes in PT ion transport thought to be mediated by microvillar bending. We found that FSS also affects apical endocytosis in PT cells. Exposure of immortalized PT cell lines to physiologically relevant levels of FSS led to dramatically increased internalization of the megalincubilin ligand albumin as well as the fluid phase marker dextran. FSS-stimulated apical endocytosis was initiated between 15 and 30 min postinduction of FSS, occurred via a clathrin- and dynamindependent pathway, and was rapidly reversed upon removing the FSS. Exposure to FSS also caused a rapid elevation in intracellular $\mathrm{Ca}^{2+}\left[\mathrm{Ca}^{2+}\right]_{i}$, which was not observed in deciliated cells, upon treatment with BAPTA-AM, or upon inclusion of apyrase in the perfusion medium. Strikingly, deciliation, BAPTA-AM, and apyrase also blocked the flow-dependent increase in endocytosis. Moreover, addition of ATP bypassed the need for FSS in enhancing endocytic capacity. Our studies suggest that increased $\left[\mathrm{Ca}^{2+}\right]_{i}$ and purinergic signaling in response to FSS-dependent ciliary bending triggers a rapid and reversible increase in apical endocytosis that contributes to the efficient retrieval of filtered proteins in the PT.
\end{abstract}

flow | calcium | ryanodine

$\mathrm{T}$ he kidney maintains stable efficient solute and fluid reabsorption over a wide range of glomerular filtration rates (GFRs), which is essential to preserve glomerulotubular balance $(1,2)$. The majority of filtered water, $\mathrm{Na}^{+}$, proteins, and other solutes are reabsorbed in the proximal tubule (PT), which plays a critical role in blood volume homeostasis. Internalization of filtered low molecular weight (LMW) proteins, vitamins, hormones, and other small molecules is mediated by the PT multiligand receptors megalin and cubilin (3). Defects in the uptake of these ligands leads to LMW proteinuria, which contributes to the pathogenesis of many renal diseases including acute and chronic kidney injury, metal toxicity, cystinosis, and the X-linked disorders Lowe syndrome and Dent disease $(4,5)$.

Increases in GFR lead to acute changes in PT ion transport capacity. The sodium-hydrogen exchanger NHE3 rapidly accumulates at the apical surface in response to the increased fluid shear stress (FSS) on PT cells to enable increased $\mathrm{Na}^{+}$reabsorption $(2,6)$. Modeling studies have suggested that these flowmediated changes in ion transport are regulated by a mechanosensitive mechanism induced by microvillar bending $(7,8)$. Increases in GFR also enhance the need for megalin-cubilinmediated uptake of filtered ligands. However, it is unknown whether or how endocytosis in PT cells responds to changes in FSS.

Here we have investigated the effect of increased flow and the accompanying FSS on apical endocytosis in PT-derived epithelial cells. We find a rapid and sustained increase in endocytic uptake of both the megalin-cubilin ligand albumin and a fluid phase marker upon exposure to physiologically relevant levels of FSS. Both basal- and FSS-stimulated uptake were inhibited by perturbants of clathrin assembly and dynamin function. Exposure to flow also triggered an increase in intracellular $\mathrm{Ca}^{2+}$ concentration $\left(\left[\mathrm{Ca}^{2+}\right]_{\mathrm{i}}\right)$ that required release of extracellular ATP and the presence of primary cilia. Importantly, deciliation of cells or inclusion of apyrase in the medium did not alter endocytosis under static conditions but completely abrogated the FSS-stimulated endocytic response. Our data suggest that flow sensing by mechanosensitive channels in the primary cilia modulates acute apical endocytic responses in PT cells. We discuss the impact of these results on our understanding of normal and disease kidney physiology.

\section{Results}

Exposure to FSS Stimulates Apical Endocytosis in PT Cells. A major function of the PT is to internalize solutes and LMW proteins from the glomerular ultrafiltrate. To this end, cells lining the PT express high levels of the multiligand receptors megalin and cubilin, and are specialized to maintain robust apical endocytic capacity (9-11). To confirm that immortalized cell models of the PT retain a high capacity for apical endocytosis, OK cells and LLC-PK1 cells were exposed to apically- or basolaterally added fluorescently tagged albumin (a megalin-cubilin ligand) and dextran (a marker for fluid phase endocytosis). As shown in Fig. $\mathrm{S} 1$, both of these cell lines internalized albumin and dextran preferentially from the apical surface. Similarly, murine S3 cells, derived from the S3 segment of the PT, also internalized albumin and dextran preferentially from the apical surface, although endocytosis was less robust than in the other PT cells (Fig. S1).

\section{Significance}

The proximal tubule (PT) of the kidney is the primary site for reabsorption of ions, solutes, and filtered low molecular weight proteins. PT cells rapidly modulate ion transport capacity in response to the fluid shear stress (FSS) that accompanies changes in glomerular filtration rate. We report here that PT cells also adjust their capacity for endocytosis in response to FSS. Apical endocytosis of the megalin-cubilin ligand albumin and of fluid phase markers is markedly increased upon exposure to FSS. Moreover, $\mathrm{Ca}^{2+}$ signaling mediated by the primary cilia on PT cells is required for this response. These studies define a novel pathway in PT cells that plays an essential role in maintaining kidney function.

Author contributions: V.R., N.M.P.-S., M.D.C., and O.A.W. designed research; V.R., Y.R. and N.M.P.-S. performed research; V.R., Y.R., N.M.P.-S., M.D.C., and O.A.W. analyzed data; and V.R. and O.A.W. wrote the paper.

The authors declare no conflict of interest.

This article is a PNAS Direct Submission.

${ }^{1}$ To whom correspondence should be addressed. E-mail: weisz@pitt.edu.

This article contains supporting information online at www.pnas.org/lookup/suppl/doi:10 1073/pnas.1402195111/-/DCSupplemental. 
As a control, we performed similar experiments in mouse cortical collecting duct (CCD) cells. These cells express little if any megalin, and not surprisingly, little albumin was internalized from either surface of these cells. However, consistent with previous studies demonstrating more efficient basolateral internalization rates compared with apical endocytosis $(12,13)$, we observed considerably greater levels of dextran internalized from the basolateral surface in these cells (Fig. S1).

Healthy individuals efficiently clear LMW proteins and other freely filtered megalin-cubilin ligands despite wide fluctuations in GFR. It is unknown whether endocytic capacity in PT cells varies with changes in fluid flow and the accompanying FSS. To test this, we asked whether changes in FSS result in increased apical endocytosis in immortalized PT cells. Polarized OK and LLC-PK1 kidney cells cultured in a parallel plate flow chamber were exposed for $3 \mathrm{~h}$ to physiologically relevant levels of FSS [1 dyne $/ \mathrm{cm}^{2}$, corresponding to a GFR of $115 \mathrm{~mL} / \mathrm{min} / 1.73 \mathrm{~m}^{2}$ (14-16)] or maintained under static conditions. The megalincubilin ligand Alexa Fluor 647-albumin $[40 \mu \mathrm{g} / \mathrm{mL}$; the reported concentration of albumin in the PT is $\sim 23 \mu \mathrm{g} / \mathrm{mL}$ (17)] or the fluid phase marker rhodamine-dextran were included in the perfusate, and the effects on receptor-mediated and fluid phase endocytosis were quantified. We reproducibly observed a striking (typically $\sim$ twofold) and statistically significant increase in the uptake of both albumin and dextran in both cell lines exposed to FSS compared with controls maintained under static conditions (Fig. 1). Identical results were obtained when the cells were cultured in Ibidi six-well slide chambers, which were used for most subsequent experiments. Because cells under FSS were exposed to a greater volume of medium than cells incubated

A

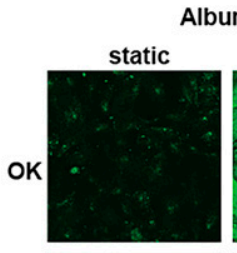

Albumin FSS
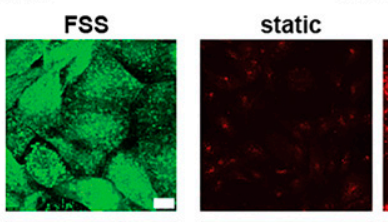
FS
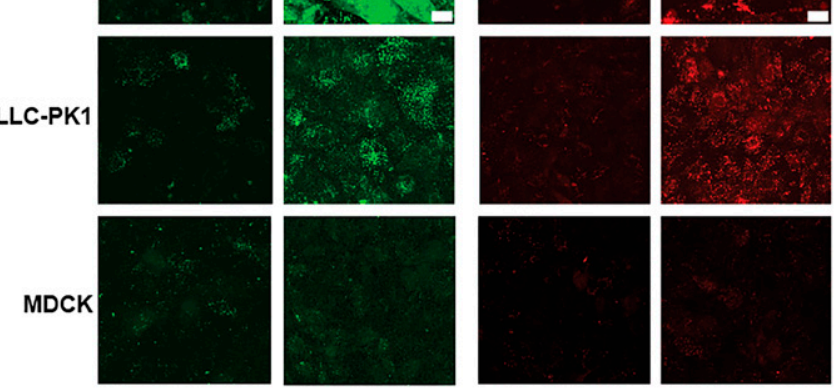

B
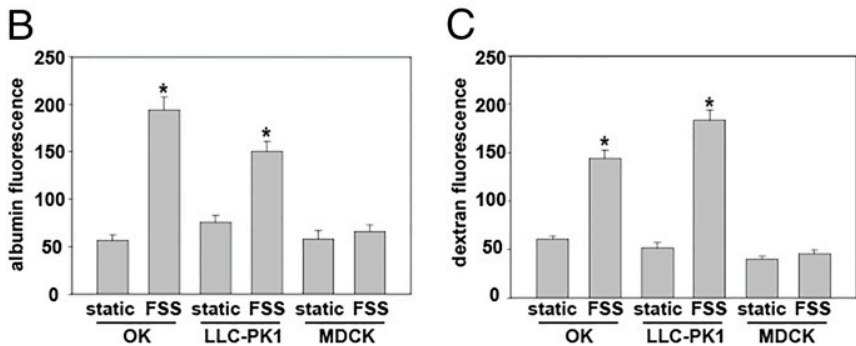

Fig. 1. Exposure to FSS increases apical albumin and dextran uptake in PT cell lines. $(A) \mathrm{OK}, \mathrm{LLC}-\mathrm{PK} 1$, or MDCK cells were incubated with $40 \mu \mathrm{g} / \mathrm{mL}$ Alexa Fluor 647-albumin and $1 \mathrm{mg} / \mathrm{mL}$ rhodamine-dextran for $3 \mathrm{~h}$ under static conditions or during exposure to $1-$ dyne $/ \mathrm{cm}^{2}$ FSS. Images of representative fields captured under identical conditions are shown. Scale bars, $10 \mu \mathrm{m}$. ( $B$ and $C$ ) The average albumin $(B)$ and dextran $(C)$ fluorescence intensity per field was quantitated in 30 fields taken from three independent experiments and the mean \pm SEM is plotted. ${ }^{*} P<0.05$ vs. static control by Student $t$ test. under static conditions, we confirmed that fluorescent albumin and dextran were not noticeably depleted from the medium under our static incubation conditions; thus, this does not account for the different in uptake that we observed (Fig. S2). Our results are consistent with those of Ferrell et al., who recently reported similar effects of FSS on albumin uptake in OK cells (18). The increase in both receptor-mediated and fluid phase uptake in PT cells upon exposure to FSS suggests a generic stimulation in endocytosis under these conditions, rather than a selective effect on megalin-cubilin trafficking. In contrast, apical endocytosis in MDCK type II cells, which have hybrid characteristics of proximal and distal tubule cells, was unaffected by FSS (Fig. 1). Thus, the FSS-stimulated increase in endocytosis is apparently selective for cells of PT origin.

Onset, Reversibility, and FSS Dependence of FSS-Stimulated Endocytosis. To test the kinetics of FSS-stimulated endocytosis, we incubated OK cells under static conditions or exposed them to $1-$ dyne $/ \mathrm{cm}^{2}$ FSS in the presence of fluorescent albumin for various time points, then fixed and quantified uptake. As shown in Fig. 2A, uptake rates under FSS and static conditions were similar for the first $15 \mathrm{~min}$ and then diverged. By $30 \mathrm{~min}$ of exposure to flow, there was a statistically significant increase in the overall amount of albumin internalized compared with cells treated under static conditions. This increased rate of uptake continued linearly for the remaining incubation period (Fig. $2 A$ ). In separate experiments, we monitored uptake over a 1-3-h incubation period (Fig. $2 A$, Inset). Uptake of albumin in static and FSS-treated cells remained linear over this time period, with a roughly twofold increase in endocytosis in FSS-treated cells compared with static at each time point.

We next measured the reversibility of the FSS-stimulated increase in endocytosis. To this end, we exposed cells to FSS for $1 \mathrm{~h}$ in the presence (Fig. 2B, sample 1) or absence (Fig. $2 B$, samples 2-4) of added albumin, then stopped the flow for various times (15-60 min; Fig. 2B, samples 2-4) before adding fluorescent albumin to the cells for $1 \mathrm{~h}$ under static conditions. As controls, we incubated cells with fluorescent albumin for a 1-h period in the absence of flow either at the start of the experiment (at $0^{\prime}$; Fig. 2B, sample 5) or coincident with the last uptake period (starting at $2 \mathrm{~h}$; Fig. $2 B$, sample 6). Albumin uptake in the presence of flow was robust compared with cells not exposed to FSS (Fig. 2B; compare sample 1 to samples 5 and 6). Strikingly, reversal of the endocytic response to FSS was essentially complete by $15 \mathrm{~min}$ after the cessation of FSS (Fig. 2B, sample 2).

In humans, normal GFR varies between 60 and $120 \mathrm{~mL} / \mathrm{min} /$ $1.73 \mathrm{~m}^{2}$ but can reach $160 \mathrm{~mL} / \mathrm{min} / 1.73 \mathrm{~m}^{2}$ in diseased individuals. To test whether PT cells adjust their endocytic capacity in response to changes in FSS induced within this range, we quantified the internalization of Alexa Fluor 647-albumin in OK cells exposed to FSS within a range of $0-1.5 \mathrm{dyne} / \mathrm{cm}^{2}$ for $1 \mathrm{~h}$. As shown in Fig. $2 C$, there was a general increasing trend in the amount of Alexa Fluor 647-albumin internalized by cells exposed to this range of FSS. Albumin internalization did not reach statistical significance relative to static controls $\left(0 \mathrm{dyne} / \mathrm{cm}^{2}\right)$ at $0.2,0.4$, and $0.7 \mathrm{dyne} / \mathrm{cm}^{2}$, but was significantly different in cells exposed to FSS of $1.0 \mathrm{dyne} / \mathrm{cm}^{2}$ and above. Importantly, there was a statistically significant increase in albumin uptake at cells exposed to 1.0 and 1.5 dyne $/ \mathrm{cm}^{2}$ compared with 0.7 dyne $/ \mathrm{cm}^{2}$ (equivalent to a GFR of $\sim 60 \mathrm{~mL} / \mathrm{min} / 1.73 \mathrm{~m}^{2}$ ). Exposure to higher FSS (1.5 dyne $/ \mathrm{cm}^{2}$, equivalent to a GFR of $\sim 150 \mathrm{~mL} / \mathrm{min} / 1.73 \mathrm{~m}^{2}$ ) did not increase endocytic capacity above the level observed at 1.0 dyne $/ \mathrm{cm}^{2}$ (Fig. $2 C$ ). This suggests that PT cells tune their internalization to maximum capacity in response to altered GFR within the normal physiologic range.

FSS-Stimulated Endocytosis Occurs via a Clathrin- and DynaminDependent Pathway. Megalin is internalized into clathrin-coated pits that form at the base of microvilli of PT cells $(10,19)$. Although some immortalized PT cell lines express caveolin, caveolae are absent in PT cells in vivo (20), suggesting that clathrin-dependent endocytosis represents the primary mechanism for internalization of 

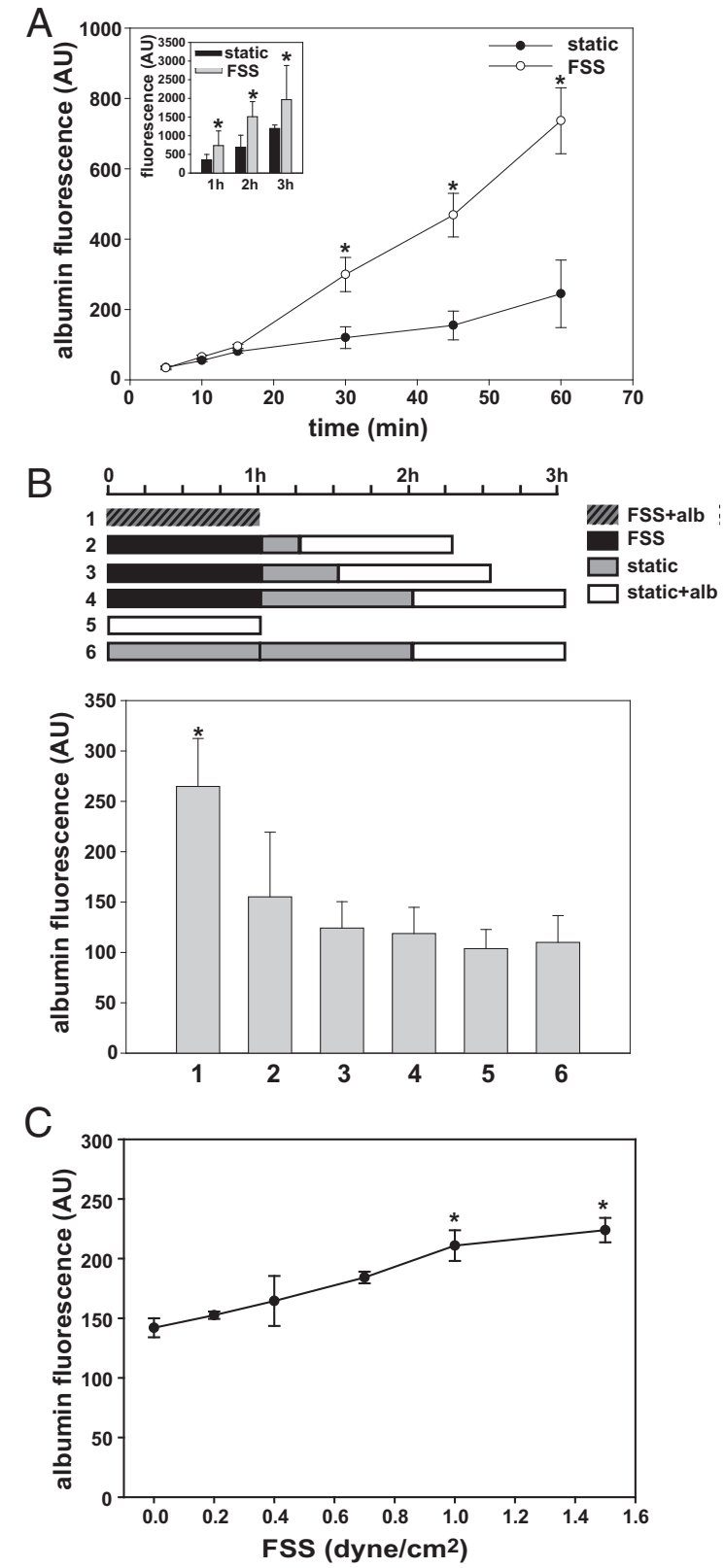

Fig. 2. Time course, reversibility, and FSS threshold of FSS-stimulated apical endocytosis. $(A)$ Time course of onset of FSS-stimulated endocytosis. OK cells plated in Ibidi $\mu$-slide chambers were incubated under static conditions or exposed to 1-dyne $/ \mathrm{cm}^{2}$ FSS in the presence of $40 \mu \mathrm{g} / \mathrm{mL}$ Alex Fluor 647-albumin for the indicated time periods, then fixed, and average internalized fluorescence quantified from 15 to 20 fields per condition. ${ }^{*} P<0.04$ vs. paired static control by Student $t$ test. (Inset) Albumin uptake over a 1-3-h time course. ${ }^{*} P<0.02$ vs. static control by $t$ test. $(B)$ Reversibility of FSS-stimulated endocytosis. OK cells were exposed to 1 -dyne $/ \mathrm{cm}^{2}$ FSS for $1 \mathrm{~h}$ in the presence (1) or absence (2-4) of $40 \mu \mathrm{g} / \mathrm{mL}$ Alexa Fluor 647-albumin. Cells were then fixed immediately (1) or incubated under static conditions for $15 \mathrm{~min}$ (2), $30 \mathrm{~min}$ (3), or $60 \mathrm{~min}$ (4) before addition of $40 \mu \mathrm{g} / \mathrm{mL}$ Alex Fluor 647-albumin for $1 \mathrm{~h}$. As controls, Alexa Fluor 647-albumin was added to cells incubated under static conditions for $1 \mathrm{~h}$ at the start of the time course (5) or after $2 \mathrm{~h} \mathrm{(6)}$ to coincide with the uptake period for sample 4 . Internalized fluorescence was quantified for five fields per condition. The average fluorescence \pm range from two independent experiments is plotted. $\star P<0.05$ vs. static control (sample 6 ) by ANOVA with Bonferroni correction. All other pairwise comparisons are not significantly different. (C) OK cells were incubated with $40 \mu \mathrm{g} / \mathrm{mL}$ Alexa Fluor 647-albumin for $1 \mathrm{~h}$ under static conditions ( 0 dyne $/ \mathrm{cm}^{2}$ ) or during exposure to the indicated FSS. Average internalized fluorescence was quantified from four wells for each membrane and fluid from the apical surface of these cells. To test whether the FSS-stimulated component of albumin endocytosis occurs via a mechanism similar to that of basal uptake, we asked whether perturbants of clathrin-dependent endocytosis disrupted albumin uptake under static conditions and upon exposure to FSS. To this end, we preincubated cells for 30 min with chlorpromazine (a drug that inhibits assembly of clathrin coats) before addition of fluorescent albumin under static conditions or in the presence of $1-d y n e / \mathrm{cm}^{2}$ FSS. Treatment with chlorpromazine reproducibly and significantly inhibited both basal and FSS-stimulated endocytosis (by $42 \%$ and $33 \%$, respectively; Fig. $3 A$ ). Treatment with the dynamin in hibitor Dyngo-4a also reduced cell-associated albumin (by $49 \%$ and $62 \%$ in cells exposed to static and FSS conditions, respectively; Fig. $3 B$ ).

FSS Triggers a Cytosolic $\mathrm{Ca}^{2+}$ Response Required for Stimulated Apical Endocytosis. Modeling studies have suggested that the flow-mediated changes in ion transport are regulated by a mechanosensitive mechanism induced by microvillar bending $(7$, 8 ). There is good evidence that primary cilia are not required for this pathway, as similar effects were observed in cells lacking mature cilia (16). In contrast, primary cilia are known to play an essential role in flow-mediated regulation of ion transport in the distal tubule (21). Genetic defects that affect cilia structure or function cause kidney disease, presumably as a consequence of aberrant FSS-dependent signaling $(21,22)$. Exposure to FSS is known to activate transient receptor potential channels localized on primary cilia to trigger an increase in $\left[\mathrm{Ca}^{2+}\right]_{\mathrm{i}}$ in many cell types, including kidney CCD cells $(2,21,23)$. To test if exposure to FSS triggers a similar response in PT cells, polarized OK cells loaded with Fura-2 AM were perfused with Krebs buffer at an FSS of 2 dyne $/ \mathrm{cm}^{2}$ and the change in $\left[\mathrm{Ca}^{2+}\right]_{\mathrm{i}}$ was determined as described in Methods. Exposure to FSS caused an immediate three- to fourfold increase in $\left[\mathrm{Ca}^{2+}\right]_{\mathrm{i}}$ that returned to baseline levels in 3-4 min (Fig. 4). The FSS-stimulated increase in $\left[\mathrm{Ca}^{2+}\right]_{\mathrm{i}}$ was not observed when $\mathrm{Ca}^{2+}$ was omitted from the perfusion buffer, demonstrating a requirement for extracellular $\mathrm{Ca}^{2+}$ in this response (Fig. $4 A$ ).

To test the role of the primary cilia in the FSS-stimulated increase in $\left[\mathrm{Ca}^{2+}\right]_{i}$ we deciliated OK cells using $30 \mathrm{mM}$ ammonium sulfate for $3 \mathrm{~h}$. We previously showed that this treatment results in efficient and reversible removal of cilia (ref. 24 and Fig. $5 A$ ). As shown in Fig. $4 B,\left[\mathrm{Ca}^{2+}\right]_{\mathrm{i}}$ in deciliated cells did not increase in response to FSS.

Previous studies conducted in collecting duct cells have shown that the FSS-stimulated, cilium-dependent increase in $\left[\mathrm{Ca}^{2+}\right]_{\mathrm{i}}$ is mediated by $\mathrm{Ca}^{2+}$-stimulated $\mathrm{Ca}^{2+}$ release from the endoplasmic reticulum (ER) via ryanodine receptors (RyRs) (21). To assess the contribution of the $\mathrm{Ca}^{2+}$-stimulated $\mathrm{Ca}^{2+}$ release to FSSstimulated increase in $\left[\mathrm{Ca}^{2+}\right]_{\mathrm{i}}$, we treated OK cells with the sarcoplasmic/endoplasmic reticulum $\mathrm{Ca} 2+-\mathrm{ATPase}$ (SERCA) inhibitor tBuBHQ to deplete ER reserves of $\mathrm{Ca}^{2+}$ and then subjected them to FSS. Resting $\left[\mathrm{Ca}^{2+}\right]_{\mathrm{i}}$ in tBuBHQ-treated cells was elevated relative to untreated cells as expected, and was unaffected upon exposure to FSS, confirming that ER stores of $\mathrm{Ca}^{2+}$ contribute to the FSS-stimulated rise in $\left[\mathrm{Ca}^{2+}\right]_{i}($ Fig. $4 C)$. We then depleted the RyR-sensitive pool of $\mathrm{ER} \mathrm{Ca}^{2+}$ using ryanodine to test the role of RyRs in FSS-stimulated increase in $\left[\mathrm{Ca}^{2+}\right]_{\mathrm{i}}$. As shown in Fig. $4 C$, we observed that the flow-stimulated increase in $\left[\mathrm{Ca}^{2+}\right]_{\mathrm{i}}$ was ablated posttreatment with ryanodine, confirming that release of the RyR sensitive pool of $\mathrm{ER} \mathrm{Ca}^{2+}$ is requisite for the flow-stimulated increase in $\left[\mathrm{Ca}^{2+}\right]_{\mathrm{i}}$. Additionally, buffering cytosolic $\mathrm{Ca}^{2+}$ by incubation with the cell permeable $\mathrm{Ca}^{2+}$ chelator bis-(o-aminophenoxy)-N,N, $\mathrm{N}^{\prime}, \mathrm{N}^{\prime}$-tetraacetic acid-acetoxymethyl

time point. ${ }^{*} P<0.05$ vs. all other conditions by ANOVA, except endocytosis measured at 1.0 vs. $1.5 \mathrm{dyne} / \mathrm{cm}^{2}$ are not significantly different from each other. 

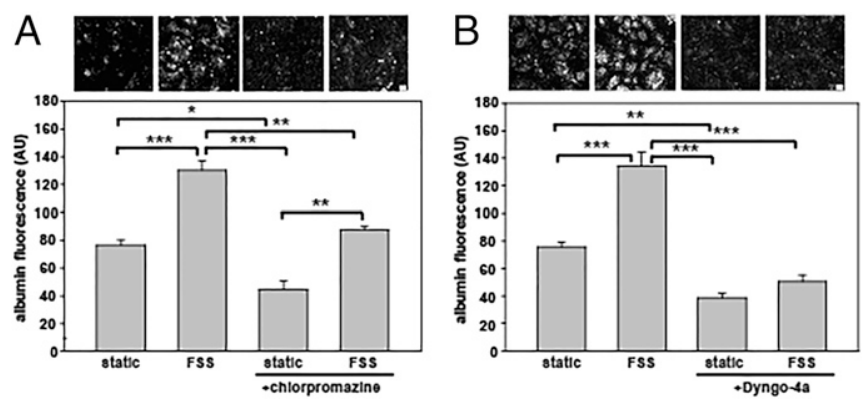

Fig. 3. FSS-stimulated uptake occurs via clathrin- and dynamin-dependent endocytosis. $(A) \mathrm{OK}$ cells cultured on Ibidi $\mu$-slide chambers were pretreated where indicated with $20 \mu \mathrm{M}$ chlorpromazine for $30 \mathrm{~min}$. Cells were exposed to $40 \mu \mathrm{g} / \mathrm{mL}$ Alexa Fluor 647-albumin for $1 \mathrm{~h}$ under static conditions or at $1-$ dyne $/ \mathrm{cm}^{2}$ (FSS). Albumin uptake was quantified as described in Methods, and the mean \pm SEM total albumin uptake in three independent experiments is plotted. $(B)$ The effect of $30 \mu \mathrm{M}$ Dyngo-4a on basal and FSS-stimulated albumin uptake was quantified from four independent experiments. Mean \pm SEM is plotted. Maximum projections of representative fields of cells are shown above each bar in the graphs. Scale bar, $10 \mu \mathrm{m}$. ${ }^{\star} P=0.014 ;{ }^{*} P \leq$ $0.005 ; * * * P<0.001$ by ANOVA with Bonferroni correction. Other pairwise comparisons are not significantly different.

(BAPTA-AM) inhibited the FSS-stimulated increase in $\left[\mathrm{Ca}^{2+}\right]$ (Fig. 4C).

Bending of the cilium in distal tubule kidney cells is also known to cause extracellular release of ATP, which can trigger activation of purinergic receptors (P2YRs) causing a further increase in $\left[\mathrm{Ca}^{2+}\right]_{\mathrm{i}}(25)$. Therefore, we tested whether depletion of extracellular ATP alters the FSS-stimulated increase in $\left[\mathrm{Ca}^{2+}\right]_{i}$ in PT cells. As shown in Fig. $4 D$, we found that inclusion of apyrase in the perfusion attenuated the FSS-stimulated increase in $\left[\mathrm{Ca}^{2+}\right]_{\mathrm{i}}$. The involvement of P2YRs in this signaling cascade was confirmed by addition of the pan P2YR inhibitor suramin, which also abrogated the FSS-stimulated $\mathrm{Ca}^{2+}$ response (Fig. $4 D$ ). Together, these data show that FSS triggers an increase in $\left[\mathrm{Ca}^{2+}\right]_{\mathrm{i}}$, which requires the primary cilium, extracellular $\mathrm{Ca}^{2+}$ influx, release of $\mathrm{Ca}^{2+}$ from ER stores through ryanodine receptors, and ATP-dependent activation of P2Y receptors.

Primary Cilia and Purinergic Signaling Are Required for FSSDependent Modulation of Endocytosis. To test whether primary cilia and the ATP-dependent $\mathrm{Ca}^{2+}$ response are also required for the endocytic response to FSS in PT cells, we deciliated OK cells as above, and measured internalization of Alexa Fluor 647-albumin in cells incubated under static conditions or exposed to 1 -dyne $/ \mathrm{cm}^{2}$ FSS. Indirect immunofluorescence confirmed that our deciliation protocol resulted in removal of essentially all primary cilia (Fig. $5 A$ ). Strikingly, whereas basal albumin uptake under static conditions was unaffected in deciliated cells, the FSS-induced increase in endocytic uptake was almost entirely abrogated (Fig. $5 A$ and $B$ ). Similarly, inclusion of BAPTA-AM (Fig. $5 C$ ) or apyrase (Fig. $5 D$ ) in the medium also blocked FSSstimulated but not basal uptake of albumin. We conclude that primary cilia and ATP-dependent P2YR signaling are both required for acute modulation of apical endocytosis in the PT in response to FSS.

Conversely, we asked whether increasing $\left[\mathrm{Ca}^{2+}\right]_{\mathrm{i}}$ in the absence of FSS is sufficient to trigger the downstream cascade that leads to enhanced endocytosis. As expected, addition of $100 \mu \mathrm{M}$ ATP in the absence of FSS caused an acute and transient threefold increase in $\left[\mathrm{Ca}^{2+}\right]_{i}$, whereas incubation with ryanodine led to a sustained elevation in $\left[\mathrm{Ca}^{2+}\right]_{\mathrm{i}}$ that was unchanged by FSS (Fig. S3A and Fig. 4C). Addition of ATP to cells incubated under static conditions also stimulated endocytosis by roughly $50 \%$ (Fig. S3B). Both basal and ATP-stimulated endocytosis were profoundly inhibited by suramin (Fig. S3B). Ryanodine also stimulated endocytosis in the absence of FSS, and this effect was not further augmented by exposure of the cells to FSS (Fig. S3C).

\section{Discussion}

PT cells are specialized to internalize and recycle large amounts of apical membrane to effectively clear LMW proteins and other molecules from the glomerular ultrafiltrate. Defects in megalin or cubilin, which mediate the uptake of these filtered ligands (3), or saturation of this pathway as can occur in diabetic nephropathy (26), lead to tubular proteinuria and eventually to renal failure. However, surprisingly little is known about how apical endocytosis is regulated in PT cells, and whether this pathway can respond acutely to variations in glomerular filtration rate to maximize uptake efficiency of filtered ligands.

Studies conducted in the past decade have underscored the importance of FSS in affecting the organization of the cytoskeleton, activity of certain transcription factors, and the trafficking and retention of transporters that regulate vectorial ion transport in PTs $(2,6)$. Our studies here demonstrate an additional role for FSS in the modulation of apical endocytosis in the PT. From our experiments we conclude that: $(i)$ exposure to FSS increases apical endocytic uptake of fluid phase and membrane-
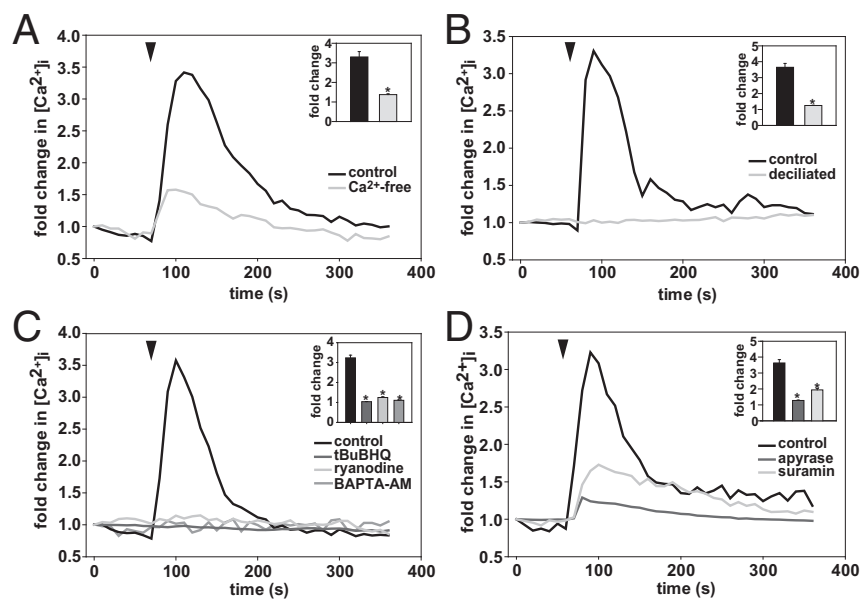

Fig. 4. Exposure to FSS causes a transient increase in $\left[\mathrm{Ca}^{2+}\right]_{i}$ that requires cilia, purinergic receptor signaling, and release of $\mathrm{Ca}^{2+}$ stores from the endoplasmic reticulum. OK cells were loaded with Fura-2 AM and $\left[\mathrm{Ca}^{2+}\right]_{\mathrm{i}}$ measured upon exposure to $2-d y n e / \mathrm{cm}^{2}$ FSS. (A) FSS stimulates a rapid increase in $\left[\mathrm{Ca}^{2+}\right]_{i}$ and this response requires extracellular $\mathrm{Ca}^{2+}$. Fura-2 AMloaded cells were perfused with $\mathrm{Ca}^{2+}$-containing (control, black traces in all subsequent panels) or $\mathrm{Ca}^{2+}$-free (light gray trace) buffer at $2 \mathrm{dyne} / \mathrm{cm}^{2}$. The traces show $\left[\mathrm{Ca}^{2+}\right]_{i}$ in an OK cell exposed to FSS. (Inset) Average peak fold change in $\left[\mathrm{Ca}^{2+}\right]_{i}$ from 18 control cells (three experiments) and 28 cells perfused with $\mathrm{Ca}^{2+}$-free buffer (four experiments). (B) $\left[\mathrm{Ca}^{2+}\right]_{i}$ does not increase in deciliated cells exposed to FSS. Cilia were removed from OK cells using $30 \mathrm{mM}$ ammonium sulfate, then cells were loaded with Fura-2 AM and subjected to FSS (light gray trace). (Inset) Average peak fold change in $\left[\mathrm{Ca}^{2+}\right]_{\mathrm{i}}$ of 18 control (three experiments) and 39 deciliated cells (four experiments). (C) The $\mathrm{Ca}^{2+}$ response requires $\mathrm{Ca}^{2+}$ release from ryanodine-sensitive ER stores. Fura-2 AM-loaded cells were treated with the SERCA inhibitor tBuBHQ (10 $\mu \mathrm{M}$; dark gray trace), BAPTA-AM (10 $\mu \mathrm{M}$; medium gray trace), or ryanodine $(25 \mu \mathrm{M}$, light gray trace). (Inset) Average peak fold change in $\left[\mathrm{Ca}^{2+}\right]_{\mathrm{i}}$ from 29 control (five experiments), 36 tBuBHQ-treated (four experiments), 47 BAPTA-AM-treated (three experiments), and 40 ryanodine-treated cells (five experiments). (D) The $\mathrm{Ca}^{2+}$ response requires extracellular ATPmediated purinergic signaling. Fura-2 AM-loaded cells were perfused with buffer containing $1 \mathrm{U} / \mathrm{mL}$ apyrase (dark gray trace) or were treated with suramin $(200 \mu \mathrm{M}$, light gray trace). (Insets) Observations from 24 control cells (four experiments), 48 cells perfused with apyrase (five experiments), and 24 suramin treated cells (four experiments). (Insets) Error bars show mean \pm SEM of the peak fold change in $\left[\mathrm{Ca}^{2+}\right]_{i}$ responses for each condition and $* P<$ 0.001 by rank-sum test. 

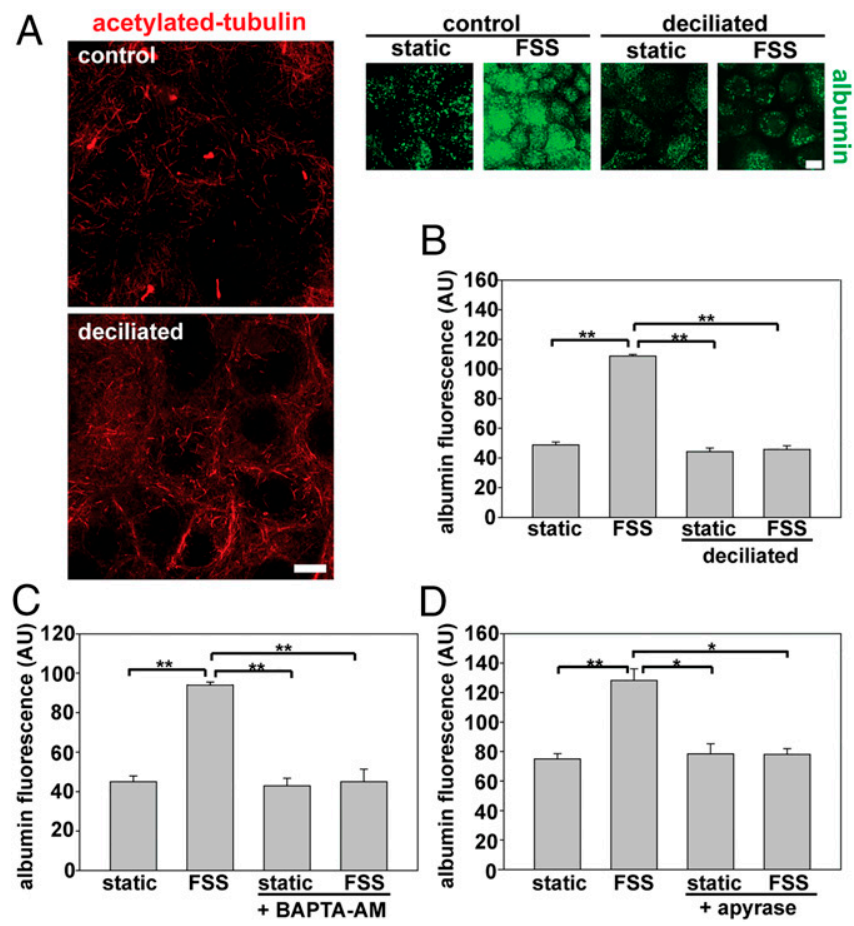

B

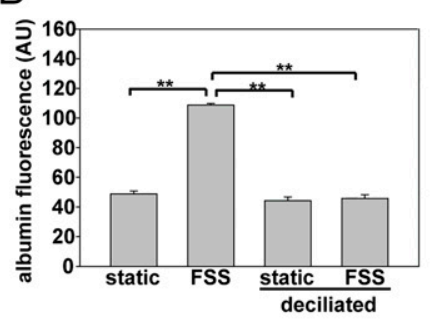

$\mathrm{D}$

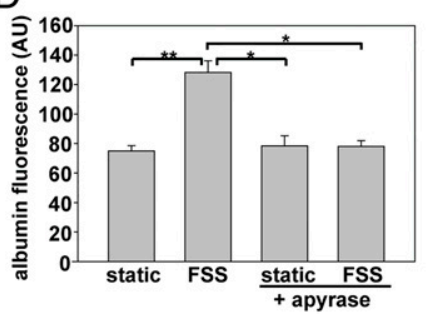

Fig. 5. FSS-stimulated apical endocytosis requires cilia and extracellular ATP. (A) OK cells were treated with ammonium sulfate as indicated to deciliate cells, then incubated with Alexa Fluor 647-albumin under static conditions or exposed to FSS $\left(1\right.$ dyne $\left./ \mathrm{cm}^{2}\right)$ for $3 \mathrm{~h}$. Cells were fixed and processed to detect cilia (with antiacetylated tubulin antibody; red) and internalized albumin (green); maximum projections of confocal stacks are shown. Scale bars, $10 \mu \mathrm{m}$. Quantitation of albumin uptake in control vs. deciliated cells $[(B)$, mean \pm SEM of three experiments], or in cells treated with $10 \mu \mathrm{M}$ BAPTA-AM $[(C)$, mean \pm SEM of four experiments] or $1 \mathrm{U} / \mathrm{mL}$ apyrase $[(D)$, mean \pm SEM of three experiments] incubated under static conditions or exposed to 1 -dyne/cm ${ }^{2}$ FSS for $1 \mathrm{~h}$. ${ }^{*} P<0.002 ; * * P<0.001$ by ANOVA with Bonferroni correction. Other pairwise comparisons are not significantly different.

bound cargoes in immortalized PT cells in culture as well as in mouse kidney slices; (ii) the FSS-stimulated endocytic response is rapid, reversible, and is mediated by a clathrin- and dynamindependent pathway; (iii) FSS also stimulates an immediate spike in intracellular $\mathrm{Ca}^{2+}$ mediated by $\mathrm{Ca}^{2+}$-dependent $\mathrm{Ca}^{2+}$ release from ER stores; $(i v)$ the primary cilium of PT cells is the principal mechanotransducer mediating the spike in FSS-stimulated intracellular $\mathrm{Ca}^{2+}$ and the subsequent endocytic response; and (v) release of extracellular ATP triggered by the bending of primary cilia in the presence of flow is required for activation of P2YRs and for FSS-stimulated endocytic responses in PT cells. A working model for how this signaling cascade might modulate endocytic capacity is shown in Fig. 6.

We observed a dramatic increase in the rate and capacity of internalization of both membrane and fluid phase markers in several immortalized PT model cell lines, suggesting that exposure to FSS triggers a generic increase in membrane and fluid uptake capacity. In contrast, apical endocytosis in a cell line with characteristics of the distal tubule was not altered by exposure to FSS. A recent study also reported a similar effect on albumin uptake in OK cells cultured in a microfluidic chamber and exposed to FSS (18). Additionally, we observed that PT cells in mouse kidney slices exposed to FSS also internalized greater levels of fluorescent dextran compared with slices incubated under static conditions. Both basal and flow-stimulated uptake in OK cells were inhibited by blockers of clathrin- and dynaminmediated endocytosis, suggesting that exposure to FSS augments the capacity of the same clathrin-dependent apical internalization pathway that operates under static conditions. Stimulation of endocytic capacity was initiated rapidly upon exposure to FSS and ended within $\sim 15$ min of removal of the FSS stimulus. Moreover, we observed a statistically significant increase in the extent of endocytosis within the normal range of FSS encountered in the PT $\left(0.7-1.0\right.$ dyne $/ \mathrm{cm}^{2}$, equivalent to GFR of $60-115 \mathrm{~mL} / \mathrm{min} / 1.73 \mathrm{~m}^{2}$ ). Indeed, endocytic capacity reached maximal levels at FSS corresponding to the upper limit of normal GFR and was not further enhanced by higher FSS, suggesting that the inability to further increase endocytic capacity may contribute to tubular proteinuria. These characteristics of the endocytic response are consistent with a physiological role for FSS-stimulated endocytosis in the PT as a mechanism to accommodate normal variations in GFR throughout the day.

Exposure of PT cells to FSS triggered an immediate increase in $\left[\mathrm{Ca}^{2+}\right]_{\mathrm{i}}$ that was not observed in the absence of the primary cilium or of extracellular $\mathrm{Ca}^{2+}$. We interpret this result to mean that $\mathrm{Ca}^{2+}$ influx mediated by a mechanosensitive channel in the cilium (likely polycystin-2) initiates the $\mathrm{Ca}^{2+}$ response to FSS Similar to cascade that has been dissected in kidney cells in the distal tubule, we found that the FSS-stimulated increase in $\left[\mathrm{Ca}^{2+}\right]$ also requires the activation of P2YRs by extracellular ATP and the release of $\mathrm{ER} \mathrm{Ca}^{2+}$ stores via the ryanodine receptor. Notably, deciliation or depletion of extracellular ATP also inhibited FSS-stimulated endocytosis in PT cells, suggesting that the increase in $\left[\mathrm{Ca}^{2+}\right]_{\mathrm{i}}$ triggered by FSS is a required step in the cascade that leads to the endocytic response. Moreover, transient or sustained elevation of $\left[\mathrm{Ca}^{2+}\right]_{\mathrm{I}}$ in the absence of FSS was sufficient to stimulate endocytic capacity.

How does initiation of the mechanotransduction cascade by FSS ultimately lead to an increase in endocytic capacity in PT cells? In principle, either an increase in the number of clathrincoated pits or an increase in the size of individual pits could account for the enhanced uptake we observed. Electron microscopy studies examining PT cells in vivo show strikingly irregular clathrin-coated invaginations at the base of apical microvilli $(9,19,27)$. Fluid phase and membrane tracers are

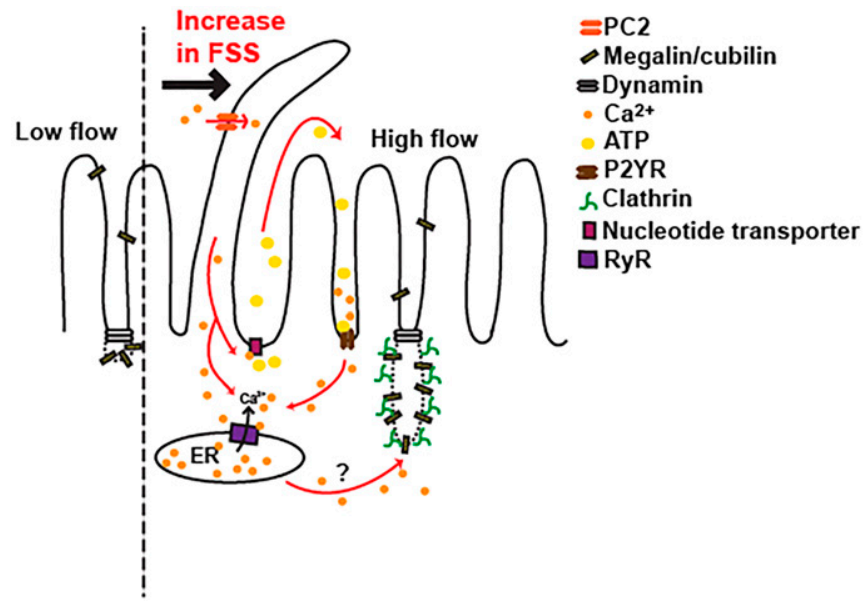

Fig. 6. Model for FSS-regulated modulation of apical endocytosis in PT. Our data support a model in which exposure to FSS increases apical endocytic capacity in PT cells via a pathway that requires ciliary bending, and entry of extracellular $\mathrm{Ca}^{2+}$ via a ciliary-localized cation channel [possibly polycystin-2 (PC2)] that lead to increases in intracellular $\mathrm{Ca}^{2+}\left(\left[\mathrm{Ca}^{2+}\right]_{i}\right)$. Bending of the primary cilium also causes release of ATP to the luminal surface (via nucleotide transporters or other mechanisms) which in turn activates P2YRs and further increases $\left[\mathrm{Ca}^{2+}\right]_{i}$. Endocytosis from the apical surface of polarized cells is known to occur exclusively at the base of microvilli via a clathrin- and dynamindependent pathway that is dependent on actin. We hypothesize that increased $\left[\mathrm{Ca}^{2+}\right]_{\mathrm{i}}$ triggers a cascade that ultimately modulates actin dynamics to increase the size and volume of individual apical clathrin-coated pits. 
internalized in these unevenly shaped structures, which bud from the apical membrane and fuse with a subapical network of tubules (19). We hypothesize that exposure to FSS increases the average size of these clathrin-coated structures to accommodate larger endocytic capacity. Consistent with this, there is precedence for modulation of clathrin-coated pit size in nonpolarized cells to accommodate larger cargoes such as virus particles (28) Unlike "traditional" clathrin-mediated endocytosis, internalization of these large cargoes requires modulation of actin dynamics at the coated pit site. We hypothesize that a similar pathway could be triggered upon FSS-stimulated $\left[\mathrm{Ca}^{2+}\right]_{\mathrm{i}}$ increases in PT cells.

The involvement of primary cilia in the endocytic response to FSS is, to our knowledge, the first known function for cilia in PT cells and raises the possibility that defects in ciliogenesis could impair the regulation of apical endocytic uptake in these cells. Genetic defects that alter ciliary function or structure lead to renal disease. To date, all disorders that result in shortened primary cilia in the kidney cause cystic disease, presumably as a consequence of aberrant flow-dependent signaling $(21,22)$. In contrast, transient elongation of cilia has been observed during kidney development and during recovery from acute kidney injury $(29,30)$. It is unclear how changes in cilia length might impact the endocytic response to FSS in the PT. Strikingly, however, cilia length has been found to be altered in cells and zebrafish depleted of OCRL1, the phosphatidylinositol 5'-phosphatase defective or absent in patients with Lowe syndrome, an $\mathrm{X}$-linked disorder that results in tubular proteinuria $(24,31,32)$. Although the precise effects or mechanism are not yet clear, as both longer and shorter cilia were reported, these studies provide a tantalizing link that could explain how loss of OCRL1 function

1. Thomson SC, Blantz RC (2008) Glomerulotubular balance, tubuloglomerular feedback, and salt homeostasis. J Am Soc Nephrol 19(12):2272-2275.

2. Weinbaum S, Duan Y, Satlin LM, Wang T, Weinstein AM (2010) Mechanotransduction in the renal tubule. Am J Physiol Renal Physiol 299(6):F1220-F1236.

3. Christensen El, Birn H, Storm T, Weyer K, Nielsen R (2012) Endocytic receptors in the renal proximal tubule. Physiology (Bethesda) 27(4):223-236.

4. Nielsen R, Christensen El (2010) Proteinuria and events beyond the slit. Pediatr Nephrol 25(5):813-822.

5. Schurman SJ, Scheinman SJ (2009) Inherited cerebrorenal syndromes. Nat Rev Nephrol 5(9):529-538.

6. McDonough AA (2010) Mechanisms of proximal tubule sodium transport regulation that link extracellular fluid volume and blood pressure. Am J Physiol Regul Integr Comp Physiol 298(4):R851-R861.

7. Du Z, et al. (2004) Mechanosensory function of microvilli of the kidney proximal tubule. Proc Natl Acad Sci USA 101(35):13068-13073.

8. Du Z, et al. (2006) Axial flow modulates proximal tubule NHE3 and H-ATPase activities by changing microvillus bending moments. Am J Physiol Renal Physiol 290(2): F289-F296.

9. Birn H, Christensen El, Nielsen S (1993) Kinetics of endocytosis in renal proximal tubule studied with ruthenium red as membrane marker. Am J Physio/ 264(2 Pt 2):F239-F250.

10. Rodman JS, Kerjaschki D, Merisko E, Farquhar MG (1984) Presence of an extensive clathrin coat on the apical plasmalemma of the rat kidney proximal tubule cell. $J$ Cell Biol 98(5):1630-1636.

11. Mattila PE, Raghavan V, Rbaibi Y, Baty CJ, Weisz OA (2014) Rab11a-positive compartments in proximal tubule cells sort fluid phase and membrane cargo. Am J Physiol Cell Physiol 306(5):C441-C449.

12. Bomsel M, Prydz K, Parton RG, Gruenberg J, Simons K (1989) Endocytosis in filtergrown Madin-Darby canine kidney cells. J Cell Biol 109(6 Pt 2):3243-3258.

13. Szalinski CM, et al. (2013) PIP5KIß selectively modulates apical endocytosis in polarized renal epithelial cells. PLOS ONE 8(1):e53790.

14. Chou CL, Marsh DJ (1987) Measurement of flow rate in rat proximal tubules with a nonobstructing optical method. Am J Physiol 253(2 Pt 2):F366-F371.

15. Maunsbach $A B$, Giebisch $G H$, Stanton $B A$ (1987) Effects of flow rate on proximal tubule ultrastructure. Am J Physiol 253(3 Pt 2):F582-F587.

16. Duan $Y$, et al. (2008) Shear-induced reorganization of renal proximal tubule cell actin cytoskeleton and apical junctional complexes. Proc Natl Acad Sci USA 105(32): 11418-11423.

17. Gekle M (2005) Renal tubule albumin transport. Annu Rev Physiol 67:573-594. leads to tubular proteinuria in Lowe syndrome patients. Future studies will be required to examine whether defects in the modulation of endocytosis by FSS contribute to kidney disease in these and other patients with tubular proteinuria.

\section{Methods}

Quantitation of Flow-Dependent Endocytosis. OK cells were cultured on Ibidi six-well chambers as described above. $\mu$-Slide Luer 0.1 elbows were used to connect the chambers to a syringe pump (Harvard Apparatus). Twentymilliliter syringes were used to perfuse individual wells with $40 \mu \mathrm{g} / \mathrm{mL}$ Alexa Fluor $647-B S A$ and/or $1 \mathrm{mg} / \mathrm{mL}$ lysine-fixable rhodamine-dextran at the indicated FSS. Cells were perfused or maintained under static conditions for $1 \mathrm{~h}$ at $37{ }^{\circ} \mathrm{C}$ unless otherwise indicated. Cells were fixed with $4 \%$ paraformaldehyde or periodate-lysine-paraformaldehyde (for experiments using dextran) for $20 \mathrm{~min}$ at ambient temperature, the fix was removed, and $50 \mu \mathrm{L}$ of ProlongGold (Invitrogen) was added to each well. The chambers were imaged the next day using a Leica TCS SP5 confocal microscope. Five to ten confocal stacks of randomly selected fields were acquired per well. Images were exported as 8-bit tagged image file formats or Leica image files and analyzed using Fiji or Image J. Maximum intensity projections of stacks were obtained for each field and total intensity was calculated using the Measure function. Measurements for background were digitally subtracted from the total intensity of each field. No normalization was used, as raw values were generally reproducible across independent experiments. More details on other methods used are provided in SI Methods.

ACKNOWLEDGMENTS. We acknowledge Dr. Catherine Baty for many helpful discussions. This work was funded by grants from the National Institutes of Health (NIH; R01-DK064613 and DK54407), the Lowe Syndrome Association, and Dialysis Clinic, Inc. (to O.A.W.); NIH R01-DK084060 (to M.D.C.); and NIH R01-DK084184 (to N.M.P.-S.). We are grateful for technical support from the morphology and physiology cores of the Pittsburgh Center for Kidney Research (P30-DK079307).

18. Ferrell N, Ricci KB, Groszek J, Marmerstein JT, Fissell WH (2012) Albumin handling by renal tubular epithelial cells in a microfluidic bioreactor. Biotechnol Bioeng 109(3): 797-803.

19. Rodman JS, Seidman L, Farquhar MG (1986) The membrane composition of coated pits, microvilli, endosomes, and lysosomes is distinctive in the rat kidney proximal tubule cell. J Cell Biol 102(1):77-87.

20. Zhuang Z, Marshansky V, Breton S, Brown D (2011) Is caveolin involved in normal proximal tubule function? Presence in model PT systems but absence in situ. Am J Physiol Renal Physiol 300(1):F199-F206.

21. Nauli SM, et al. (2003) Polycystins 1 and 2 mediate mechanosensation in the primary cilium of kidney cells. Nat Genet 33(2):129-137.

22. Yoder BK (2007) Role of primary cilia in the pathogenesis of polycystic kidney disease. J Am Soc Nephrol 18(5):1381-1388.

23. Liu W, et al. (2003) Effect of flow and stretch on the [Ca2+]i response of principal and intercalated cells in cortical collecting duct. Am J Physiol Renal Physiol 285(5): F998-F1012.

24. Rbaibi Y, et al. (2012) OCRL1 modulates cilia length in renal epithelial cells. Traffic 13(9):1295-1305.

25. Praetorius HA, Leipziger J (2013) Primary cilium-dependent sensing of urinary flow and paracrine purinergic signaling. Semin Cell Dev Biol 24(1):3-10.

26. Tojo A, et al. (2001) Reduced albumin reabsorption in the proximal tubule of earlystage diabetic rats. Histochem Cell Biol 116(3):269-276

27. Hatae T, Ichimura T, Ishida T, Sakurai T (1997) Apical tubular network in the rat kidney proximal tubule cells studied by thick-section and scanning electron microscopy. Cell Tissue Res 288(2):317-325.

28. Cureton DK, Massol RH, Saffarian S, Kirchhausen TL, Whelan SP (2009) Vesicular stomatitis virus enters cells through vesicles incompletely coated with clathrin that depend upon actin for internalization. PLoS Pathog 5(4):e1000394.

29. Liu W, et al. (2005) Mechanoregulation of intracellular Ca2+ concentration is attenuated in collecting duct of monocilium-impaired orpk mice. Am J Physiol Renal Physiol 289(5):F978-F988.

30. Verghese $E$, et al. (2009) Renal primary cilia lengthen after acute tubular necrosis. J Am Soc Nephrol 20(10):2147-2153.

31. Coon BG, et al. (2012) The Lowe syndrome protein OCRL1 is involved in primary cilia assembly. Hum Mol Genet 21(8):1835-1847.

32. Luo N, et al. (2012) OCRL localizes to the primary cilium: A new role for cilia in Lowe syndrome. Hum Mol Genet 21(15):3333-3344. 